\title{
Bei neuropathischen Schmerzen: am besten Kombinationstherapie
}

\begin{abstract}
Medikamente zur Behandlung neuropathischer Schmerzen sind häufig unzureichend wirksam und mit Nebenwirkungen belastet. Die Kombinationstherapie mit Gabapentin und Nortriptylin ist besser als eine Monotherapie.
\end{abstract}

- In eine doppelblinde Cross-overStudie mit "Double-Dummy-Technik“ wurden 56 Patienten mit diabetischer oder postherpetischer Neuropathie und Schmerzen mindestens Grad 4 (auf einer Skala von 0-10) aufgenommen. In randomisierter Reihenfolge erhielten alle Patienten für die Dauer von jeweils sechs Wochen nacheinander entweder Kapseln mit 400 mg Gabapentin, 10 mg Nortriptylin oder deren Kombination. Dosissteigerungen erfolgten bis zum Auftreten von Nebenwirkungen bzw. auf Maximaldosen von $3600 \mathrm{mg}$ bzw. $100 \mathrm{mg}$. Die maxi- mal tolerierten Tagesdosen bei Monound Kombinationstherapie waren 2433 bzw. $2180 \mathrm{mg}$ für Gabapentin und 61,6 bzw. 50,1 mg für Nortriptylin.

Die Schmerzen gingen von Grad 5,4 vor Therapie auf 3,2 nach Gabapentin, 2,9 nach Nortriptylin und 2,3 nach Kombinationstherapie - signifikant gegenüber beiden Monotherapien zurück. Die häufigste Nebenwirkung in allen Behandlungsarmen war Mundtrockenheit, allerdings signifikant seltener unter Gabapentin als unter Nortriptylin und Kombinationstherapie. Ernsthafte Nebenwirkungen traten nicht auf.

KOMMENTAR: Kombinationstherapie ist ein anerkanntes und bewährtes Prinzip, z.B. bei der Behandlung maligner Erkrankungen und der Hypertonie. Dabei geht es nicht allein um Wirkungsverstärkung, sondern auch darum, multiple pathogenetische Mechanismen differenziert zu attackieren. Auch Schmerzen haben häufig multifaktorielle Ursachen und werden von den Patienten individuell verarbeitet. Deshalb bietet sich auch bei neuropathischen Schmerzen eine Kombinationstherapie an. Offen bleibt, ob zunächst immer eine Monotherapie eingeleitet und erst bei unzureichender Wirkung eine Kombinationstherapie folgen soll, oder ob die Kombination schon am Anfang stehen soll. Die Autoren beschreiben eine erhöhte Wirksamkeit der Kombination ohne Zunahme der Nebenwirkungen, d.h. die erwünschten Wirkungen addieren sich stärker als die unerwünschten.

H. HOLZGREVE .

\section{- I. Gilron et al.}

Nortriptyline and gabapentin, alone and in combination for neuropathic pain; a doubleblind, randomised controlled crossover trial. Lancet 374 (2009) 1252-1261

\section{Am Herzen lag's nicht}

\section{Neue Ursache für Uhrglasnägel}

— Der 43-jährige Mann erlitt eine lazerierende Verletzung im Bereich des linken Handgelenks mit Verletzung des Nervus medianus und der Sehne des Musculus flexor carpi ulnaris. Postoperativ fiel bei dem Patienten auf, dass sich im Versorgungsgebiet des linken Nervus medianus am Daumen, Zeigefinger und Mittelfinger Uhrglasnägel ausbildeten.

Trommelschlägelfinger und Uhrglasnägel sind bei Erkrankungen des kardiorespiratorischen und gastrointestinalen Systems gut bekannt, wobei sie allerdings beidseitig auftreten. Das einseitige Auftreten im Bereich des Versorgungsge-

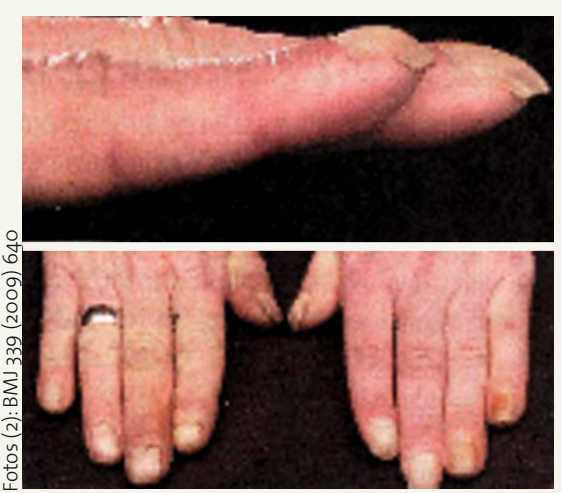

Uhrglasnägel nach Verletzung des Nervus medianus.

biets eines lädierten Nerven weist auf die Pathogenese dieses Phänomens hin, da es im Rahmen eines gestörten neu- rozirkulatorischen Reflexes zur Ausbildung eines verstärkten Blutflusses über multiple arteriovenöse Shunts in den distalen Phalangen kommt. Dies führt zu einer Hypertrophie und Hyperplasie des Weichteilgewebes. Ähnliche Mechanismen spielen bei Verletzungen des Plexus brachialis, bei der Schultersubluxation, beim Pancoasttumor, bei der Sarkoidose und bei der Herpesinfektion im Bereich der Finger eine Rolle.

H. S. FÜEßL

- D. O'Halloran, J. Webb

(Department of plastic surgery, Selly Oak Hospital, Birmingham B29 6JD). Brit. Med. J. 339 (2009) 7721,640 